\title{
PARAMETRISATION OF A NUMERICAL MODEL FOR A PARTIAL HEATING STRATEGY USED TO EVALUATE A MASKING CONCEPT OF A HOT STAMPING PROCESS
}

\author{
Bernd-Arno BEHRENS, Alexander CHUGREEV, Masood JALANESH, Kai WÖLKI, \\ Florian BOHNE \\ Leibniz Universität Hannover, Institute of Forming Technology and Machines, Germany, EU, \\ bohne@ifum.uni-hannover.de
}

https://doi.org/10.37904/metal.2019.684

\begin{abstract}
Hot stamping has become an established technology for the production of high strength steel parts in the automotive industry. The sheets are heated up to temperatures over austenitization temperature and held in order to obtain a fully austenised microstructure, formed and subsequently quenched. A locally optimized timetemperature-profile during heating provides the possibility to produce tailored parts, with locally varying microstructure and thus locally adapted mechanical properties. In order to determine an appropriate partial heating strategy, the heating as well as the cooling process has to be analyzed. In this paper a numerical model of a partial heating process is investigated in LS-DYNA comprising the heating process as well as the subsequent cooling phase. In order to describe the heating process, a new material model in LS-DYNA is used, which is suited for modeling arbitrary phase transformation processes of multiple phases with help of different transformation equations. The parameters of the numerical model are determined by means of experimental tests. The numerical findings are validated by comparison with experimental masking test.
\end{abstract}

Keywords: Hot Stamping, Partial Heating, FEM, Material Characterization, Austenitization

\section{INTRODUCTION}

The objective of reducing the vehicle mass by maintaining the passengers safety has lead to an increasing use of high strength materials. The hot stamping process is a widely known and well researched forming process, in which the sheet metal is heated over the austenitization temperature and subsequently formed and quenched. The austenitic microstructure, which is formed during the heating process transforms into the significantly harder martensitic microstructure during the cooling process. Thereby, a high strength component is obtained, which withstands extreme load cases arising in crash situations. By tailoring the local strength of a component to such a specific load case, superfluous mass can be avoided. In case of the hot stamping process locally varying properties can be obtained by the application of a partial heating strategy.

\subsection{Partial heating}

Several approaches for the adjustment of a locally adapted microstructure have been developed in the last decades. In the partial heating process the austenitization is limited to the areas, in which a microstructure transformation during the subsequent cooling process is intended. In [1] the application of a masking concept during the heat treatment is explained. The described masking is composed by a shell structure. The shell structure covers the area, which is supposed to feature a soft microstructure. Moreover, suitable materials for the masking are proposed. Based on the research a simulation model was developed to properly predict the mechanical properties caused by the hot stamping process. This provided the possibility to adapt the masking regarding the intended mechanical profile. In [2] different masking concepts, comprising different materials and thicknesses were investigated. The experimental tests were supported by numerical analysis of the heating and subsequent quenching process. 


\subsection{Modeling the hot stamping process}

For the simulation of a hot stamping process the multiple interrelations between the mechanical and thermal field as well as the microstructure have to be taken into account. The temperature profile during the process affects the formation of microstructure and thereby the resulting material strength. For conventional hot stamping analysis by means of a numerical simulation a complete austenised and homogeneous microstructure is assumed after the heating process. The focus is set on the cooling phase, in which the austenite transforms to bainite, ferrite, pearlite and martensite. The diffusion induced microstructure transformation from austenite to ferrite, pearlite and bainit is predominantly described by the Kirkaldy model [3]. The diffusionless transformation from austenite to martensite is strongly temperature dependent and can be described by means of the algebraic equation, proposed by Koistinen \& Marburger [3,4]. In processes which require the consideration of the heating phase (e.g. welding processes) the transformation from the initial microstructure into austenite hast to be taken into account $[5,6]$. In [7] an equation for describing the transformation rate of the austenite fraction is proposed

$\dot{\mu}_{a}=n\left(\ln \left(\frac{\mu_{e q}}{\mu_{e q}-\mu_{a}(t)}\right)\right)^{\frac{(n-1)}{n}} \frac{\left(\mu_{e q}-\mu_{a}\right)}{\tau}$

where $t$ is the time, $\mu_{a}$ is the austenite fraction and $\mu_{e q}$ is the eutectoid fraction formed at a specific temperature. The parameter $\tau$ and $n$ define the temperature dependent transformation rate. This approach is implemented in the material models *MAT_UHS and *MAT_PHS_BMW of the simulation software LS-DYNA. Both material models are intended for modeling hot stamping processes [8]. The implemented models assume an eutectoid fraction of $\mu_{e q}=1$, which seems to be a reasonable assumption in case of high heating rates. However, for modeling partial heating processes, in which the heating rates are intentionally reduced in order to avoid the formation of austenite in the masked areas, these material models are unsuited. Therefore, in this paper the newly developed material model *MAT_GENERALIZED_PHASE_CHANGE is applied for modeling a partial heating process. This material model provides the possibility to use the generalized Johnson-Mehl-AvramiKolmogrov (JMAK) model in order to describe arbitrary transformation processes. It can be simplified to equation 1 with the advantage that a temperature dependent eutectoid fraction can be defined.

\section{MATERIAL CHARACTERISATION AND MODELLING}

In order to characterize the temperature dependent transformation behavior of the material 22MnB5 experimental heating tests have been conducted in a quenching and forming dilatometer. The experimental setup and the length change of the specimen over temperature are presented in Figure 1.
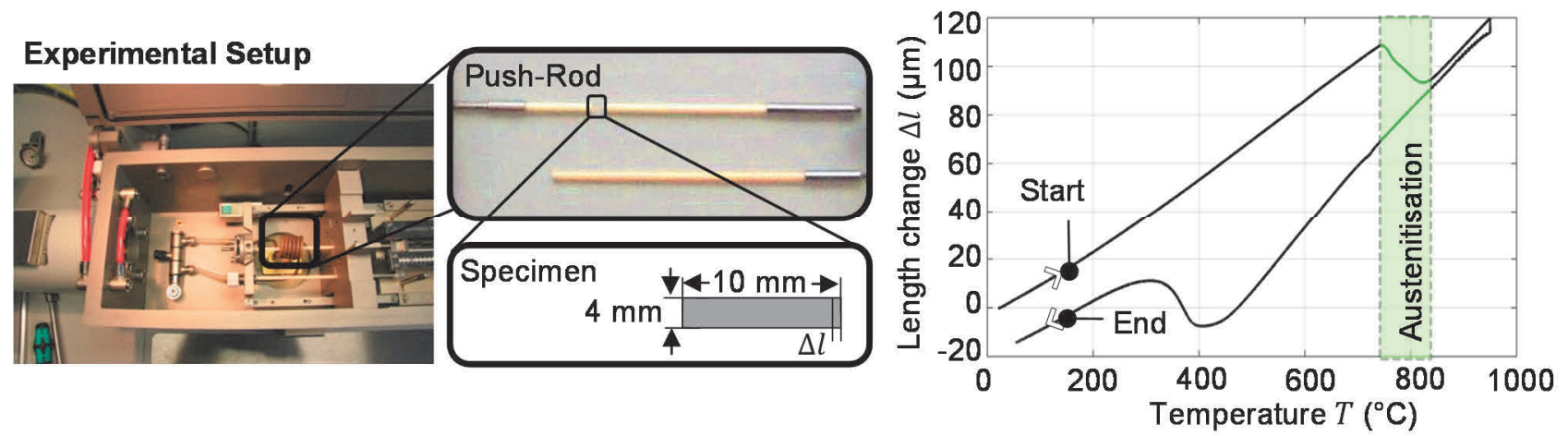

Figure 1 Experimental setup of dilatometer tests (left) and length change of the specimen over temperature (right) 
The specimens were heated by induction with a constant rate of $10 \mathrm{~K} / \mathrm{s}$ and the length change was measured. In order to parametrize the material model the fraction of austenite as function of time is required. Therefore the following approach is developed to translate the measured length change into an austenite fraction (equation 2).

$\Delta l=\int_{0}^{l_{0}} d u(X)=\int_{0}^{l_{0}} \frac{\partial u}{\partial X} d X=\int_{0}^{l_{0}} \frac{\partial(x-X)}{\partial X} d X=\int_{0}^{l_{0}}\left(\frac{\partial x}{\partial X}-1\right) d X=\left(\frac{\partial x}{\partial X}(T)-1\right) l_{0}$

Figure 2 (left) illustrates the relationship between the formation of austenite and the respective length change during heating. The change of length $\Delta l$ measured at the end of the specimen is caused by the expansion $d u$ of each element $d X$ in the reference state and can be expressed with help of an integral over the whole bar $\left(l_{0}\right.$ is the initial length). The initial position of each material point is expressed with $X$ and the actual position with $x$. It is assumed that the length of each element $d x$ can be approximated with help of a linear superposition of the normed lattice constants of the ferritic-pearlitic $\left(a_{F P}^{\prime}\right)$ and the austenitic $\left(a_{A}^{\prime}\right)$ microstructure corresponding to the actual phase fractions [4].

$d x=\left(1-\mu_{A}\right) \cdot a_{F P}^{\prime}+\mu_{A} \cdot a_{A}^{\prime} \quad d X=a_{F P}^{\prime}$

Thus the derivative of the actual location with respect to reference configuration can be expressed with help of the normed lattice constants as well as the phase fraction of austenite $\mu_{a}$.

$\frac{\partial x}{\partial X}=\frac{d x}{d X}=\frac{\left(1-\mu_{A}(T)\right) \cdot a_{F P}^{\prime}+\mu(T) \cdot a_{A}^{\prime}}{a_{F P}^{\prime}}$

Combining equation (3) with (4) and assuming that the ratio of the lattice constants can be approximated by the ratio of the specimen length before austenitization $l_{0}$ and the specimen length after austenitization $l\left(T_{A C 3}\right)$ the phase fraction can be expressed by the length change during austenitization $\Delta l(T)$ and the final length change $\Delta l\left(T_{A c 3}\right)$, after the complete microstructure is transformed. This relation has been used to calculate the phase fraction evolution within the experiment.

$\mu_{A}(T)=\frac{\Delta l(T)}{\Delta l\left(T_{A c 3}\right)}$

In order to model the austenitization process in LS-DYNA the provided material model *MAT_GENERALIZED_PHASE_CHANGE is parameterized. Therefore the implemented JMAK-equation is adapted to resemble equation (1). The JMAK-equation is given as follows [9]:

$\frac{d \mu_{A}}{d t}=n(T)\left(\frac{\mu_{e q}(T)}{\tau(T)} f(\dot{T}) \mu_{F P}-\frac{1-\mu_{e q}(T)}{\tau(T)} f^{\prime}(\dot{T}) \mu_{A}\right) \cdot\left(\ln \left(\frac{\frac{\mu_{e q}(T)}{\tau(T)} f(\dot{T})\left(\mu_{F P}+\mu_{A}\right)}{\frac{\mu_{e q}(T)}{\tau(T)} f(\dot{T}) \mu_{F P}-\frac{1-\mu_{e q}(T)}{\tau(T)} f^{\prime}(\dot{T}) \mu_{A}}\right)\right)^{\frac{n(T)-1}{n(T)}}$

For adapting this equation $f(T)$ as well as $f^{\prime}(T)$ have to be set to unity. The exponent $n(T)$ is defined as three in [7] and causes an increase of the formation rate when the phase fraction of austenite rises. In the presented analysis, this effect will be neglected, presuming that by adapting the time constant $\tau(T)$, a sufficiently precise approximation of the overall austenite formation behavior is obtained. Assuming that the austenitization process of ferrite and pearlite start at $T_{A_{C 1}}$ the following system of differential equations is obtained:

$\frac{d \mu_{F}}{d t}=\left(\frac{\mu_{e q, F \rightarrow A}(T)}{\tau\left(T, c_{1}, c_{2}\right)} \mu_{A}-\frac{1-\mu_{e q, F \rightarrow A}(T)}{\tau\left(T, c_{1}, c_{2}\right)} \mu_{F}\right)$

$\frac{d \mu_{P}}{d t}=\left(\frac{\mu_{e q, P \rightarrow A}(T)}{\tau\left(T, c_{1}, c_{2}\right)} \mu_{A}-\frac{1-\mu_{e q, P \rightarrow A}(T)}{\tau\left(T, c_{1}, c_{2}\right)} \mu_{P}\right)$

$\frac{d \mu_{A}}{d t}=-\frac{d \mu_{F}}{d t}-\frac{d \mu_{P}}{d t}$

Here, $\mu_{F}$ and $\mu_{P}$ are the phase fractions of ferrit and perlit, respectively. The eutectoid fractions are defined as follows:

$\mu_{e q, P \rightarrow A}(T)=1 \quad$ and $\quad \mu_{e q, F \rightarrow A}(T)=\frac{T-T_{A c 1}}{T_{A c 3}-T_{A c 1}}$ 
These definitions lead to the following transformation behavior [10]:

- $\quad$ pearlite transforms completely to austenite if the process temperature exceed $T_{A_{c 1}}$ and $\Delta t \rightarrow \infty$

- ferrite transforms to the temperature dependent eutectoid fraction if $\Delta t \rightarrow \infty$

The start temperature $T_{A_{C 1}}$ and the end temperatures $T_{A_{C 3}}$ for the austenitization process are defined based on the experimental data. In order to parameterize the presented model the coefficient $c_{1}$ and $c_{2}$ must be determined. Therefore the defined system of equation is implemented in a MATLAB-script. By fitting the curve of $\mu_{A}(t)$ to the experimental data, the coefficient $c_{1}$ and $c_{2}$ are obtained. The experimental and calculated time dependent phase fractions of austenite are presented in Figure 2 (right). The computed austenite evolution is in a good agreement with the experimental results. The determined parameters are introduced into the material model *MAT_GENERALIZED_PHASE_CHANGE. Moreover, the Kirkaldy-model for the cooling phase is included in the material model. The parameters of the Kirkaldy model have been determined based on a similar procedure using experimental and literature data. The hardness is computed by a mixing law, described in [3].
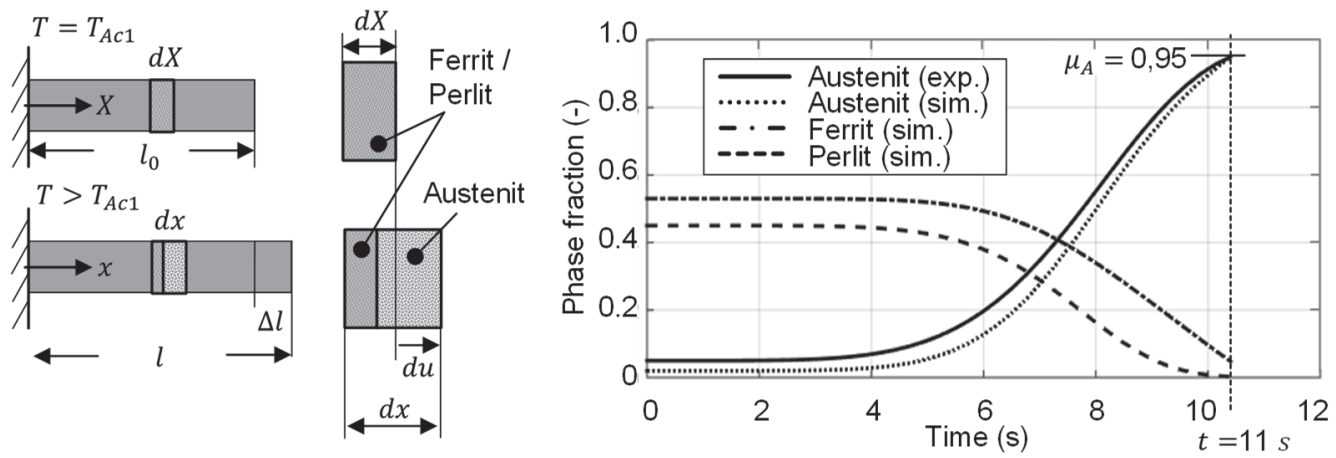

Figure 2 Schematic representation of the dilatometric test evaluation (left) and results of the parametrized transformation model defined by the equation (7) - (8) (right)

\section{SIMULATION MODEL OF THE PARTIAL HEATING PROCESS}

The obtained material model is applied to a partial heating process. The respective tests are described in [2]. A sheet consisting of $22 \mathrm{MnB} 5$ is partially covered with a mask consisting of $\mathrm{X} 2 \mathrm{CrTiMb} 18$ and heated in a furnace. After a holding time of 5 min the sheet is transferred to a press and quenched.

a)

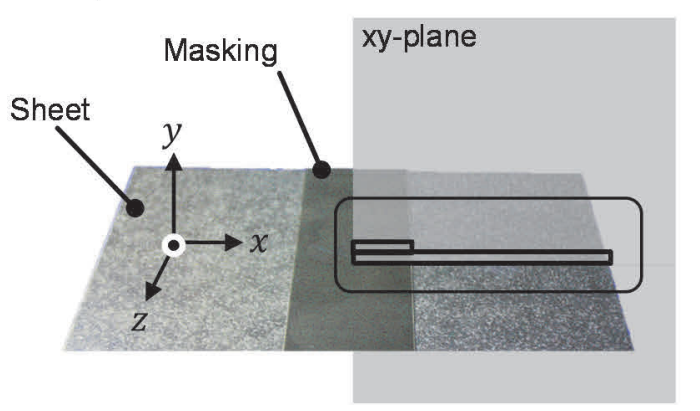

b)

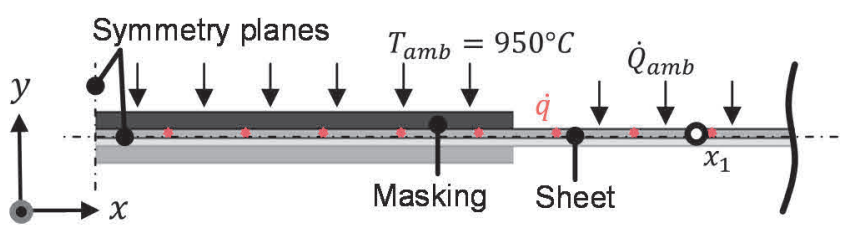

Figure 3 Experimental setup (a), temperature-time-curve of the ambient temperature $\boldsymbol{T}_{\boldsymbol{a m b} \boldsymbol{b}}$ and simulation model of the partial heating process (b)

The corresponding model is shown in Figure $\mathbf{3}$ and comprises the metallic sheet and the masking. In order to reduce computational time the model is reduced by taking advantage of symmetry planes. The furnace temperature is assumed to be $950^{\circ} \mathrm{C}$, which leads to a heat flow into the metallic sheet and the masking. 
Therefore, the heat transfer coefficients were computed based on experimental heating tests, which are described in [2]. For modelling the cooling phase a cooling rate of $40 \mathrm{~K} / \mathrm{s}$ is assumed. The cooling is introduced by heat sinks. The necessary heat consumption $\dot{q}$ is defined as a function of the temperature and can be calculated by simplifying the balance of energy for solid bodies, assuming that heat conduction is negligible during the considered cooling phase.

$\frac{\partial T}{\partial t} \rho c_{v}=\operatorname{div}(\lambda \operatorname{grad}(T))+\dot{q} \Rightarrow \frac{\partial T}{\partial t} \rho c_{v}=\dot{q}$

The heat consumption $\dot{q}$ for the cooling phase is $0.24 \mathrm{~W} / \mathrm{mm}^{3}$ and is set to zero, for all temperatures below $30^{\circ} \mathrm{C}$. This leads to a homogenous temperature distribution at the end of the cooling process to ensure that the martensite transformation has completely finished.

\section{COMPARISON OF NUMERICAL AND EXPERIMENTAL RESULTS}

The results of the simulation model and the respective experimental results [2] are presented in Figure 4. On the left of the figure the phase fraction of austenite at the end of the heating process is shown. The whole sheet is at least partially austenised, which results in the development of the martensitic microstructure after quenching. The austenite transformation in the shielded region has not been completely prevented by the mask. Moreover a transition region from totally austenised areas into partially austenised areas can be observed. It can be concluded that during the heating phase heat flows from the unshielded areas into the covered areas occurred. These heat flows cannot be avoided by the presented masking concept. Assuming that the uncovered regions underwent a conventional hot stamping process in the experiment as well as in the simulation, the phase fraction of austenite had to be one at the end of the heating process in these areas. This is properly predicted by the simulation model.
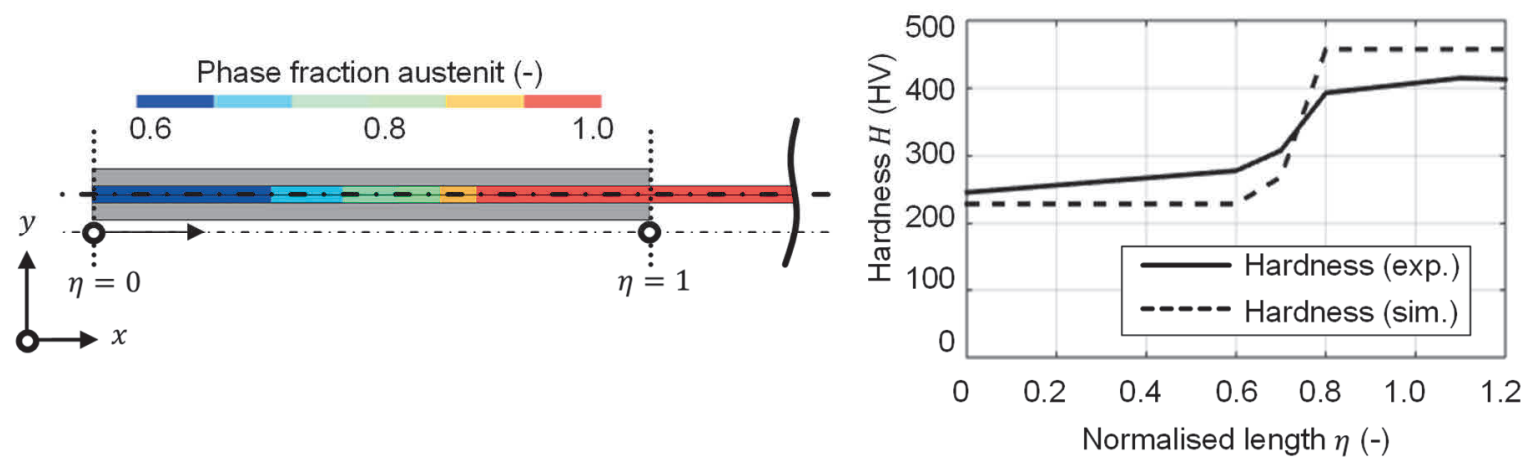

Figure 4 Calculated austenite phase fraction at the end of the heating phase (left) and comparison of experimental measured and numerical calculated final hardness distribution (right)

The hardness depends strongly on the microstructure. The comparison of the computed and experimentally measured hardness (Figure 4, right) shows a good qualitative agreement. There is a slight underestimation of the resulting hardness in the middle region of the masked area and an overestimation in the unmasked area. The differences in hardness in the middle region are assumingly caused by discrepancies in the implemented transformation law for the cooling phase. However, the deviation of hardness in the unmasked regions is probably caused by inaccuracies of the experimental measurement system. For the modelled cooling rates the computed hardness values for the unshielded area fit to the hardness of a martensitic microstructure of the steel 22MnB5.

\section{CONCLUSION}

In this work a material model for describing the austenitization process has been parametrized by means of experimental dilatometric tests and implemented into a new material model. The model has been applied to a 
partial heating process. The hardness evolution is subsequently compared to experimental hardness values and has shown a good agreement with the experimental measurements.

\section{ACKNOWLEDGEMENTS}

The presented work is a result of the project: „Partielles Formhärten durch Einsatz einer Maskierung im Ofenprozess" funded by the Deutsche Forschungsgemeinschaft - 310404283. The authors

\section{gratefully thank for the funding.}

\section{REFERENCES}

[1] EBERT, F., WÖLFER, B. and GLUECK, B. EP2264193A1: Herstellung eines partiell pressgehärteten Blechbauteils, European Patent Office, 2010.

[2] BEHRENS, B.-A., WÖLKI, K., JALANESH, M., BOHNE, F., SEEL, A., HÜBNER, S. and CHUGREEV, A. Investigation of masking concepts for influencing the austenitisation process during press hardening. Procedia Manufacturing. 2018. vol. 15, pp. 1095-1102.

[3] HOCHHOLDINGER, B. Simulation des Presshärteprozesses und Vorhersage der mechanischen Bauteileigenschaften nach dem Härten. Zürich: ETH, 2012. 236 p.

[4] BEHRENS, B.-A. and SCHRÖDTER, J. Numerical Simulation of Phase Transformation during the Hot Stamping Process. Thermal Process Modeling: Proceedings from the 5th International Conference on Thermal Process Modeling and Computer Simulation. Orlando, 2014, pp. 179-190.

[5] HENWOOD, C., BIBBY, M., GOLDAK, J. and WATT, D. Coupled transient heat transfer-microstructure weld computations (part B). Acta Metallurgica. 1988. vol. 36, no.11, pp. 3037-3046.

[6] ION, J. C., EASTERLING, K.E. and ASHBY, M.F. A second report on diagrams of microstructure and hardness for heat-affected zones in welds. Acta Metallurgica. 1984. vol. 32, no. 11, pp.1949-1955, 1957-1962.

[7] ODDY, A. S., MCDILL, J. M. J. and KARLSSON, L. Microstructural predictions including arbitrary thermal histories, reaustenization and carbon segregation effects. Canadian Metallurgical Quarterly. 1996. vol. 35, no. 3, pp. 275-283.

[8] LI, N., LIN, J., DEAN, T. A., DRY, D. and BALINT, D. Materials modelling for selective heating and press hardening of boron steel panels with graded microstructures. Procedia Engineering. 2014. vol. 81, pp. 1675 1681.

[9] LIVERMORE SOFTWARE TECHNOLOGY CORPORATION. KEYWORD USER'S MANUAL VOLUME II Material Models LS-DYNA R10.0 [online]. Last updated: 2017-07-26. 1543 p. [viewed 2019-02-01]. Available from https://www.dynasupport.com/manuals.

[10] BARGEL, H.-J. and SCHULZE, G. Werkstoffkunde [online]. 11th. ed. Springer-Verlag Berlin Heidelberg GmbH. 2012. [viewed 2018-08-13]. Available from https://www.springer.com/de/book/9783642177170. 